\section{Psychological Medicine}

cambridge.org/psm

\section{Correspondence}

Cite this article: Taylor D, Horowitz MA (2020). Antipsychotics and mortality - more clarity needed. Psychological Medicine 50, 2814-2815. https://doi.org/10.1017/S0033291720004535

Received: 2 November 2020

Revised: 4 November 2020

Accepted: 4 November 2020

First published online: 2 December 2020

Author for correspondence:

David Taylor,

E-mail: David.Taylor@slam.nhs.uk

\title{
Antipsychotics and mortality - more clarity needed
}

${\text { David } \text { Taylor }^{1} \text { (D) and Mark Abie Horowitz }}^{2}$ (D)

${ }^{1}$ King's College, London, UK and ${ }^{2}$ University College, London, UK

Nobody can fail to be intrigued by, if not sceptical of, studies which appear to show that the prescribing of antipsychotics [which can cause weight gain, metabolic disturbance, QT prolongation, akathisia-linked suicide (Salem, Nagpal, Pigott, \& Teixeira, 2017) and dose-related sudden cardiac death (Ray, Chung, Murray, Hall, \& Stein, 2009)] results in people living longer than those not prescribed these drugs. The expectation, of course, is that people taking antipsychotics live relatively short lives, and that those prescribed antipsychotics with the greatest toxic effects (such as clozapine) have amongst the highest mortality (Taylor, Douglas-Hall, Olofinjana, Whiskey, \& Thomas, 2009), not the lowest. The onus of 'proof therefore is on the researchers claiming that antipsychotic use prolongs life. Their responsibility is not so much to posit an explanation for their surprising findings, but show without doubt that their research methods and findings accurately reflect reality.

The response of Tiihonen and others (Tiihonen, Taipale, \& Correll, 2020) to the critique of Robert Whitaker (Whitaker, 2020) does not go far enough to convince us that their findings are cogent. The respondents make little attempt to rebut many of Whitaker's analyses of their studies, instead turning to discussion of short-term RCTs.

First, they do not address the main criticism of Whitaker that their method is based upon deaths occurring while patients were receiving or not receiving outpatient prescriptions for antipsychotics. Since the majority of deaths occurred in hospital, when presumably patients were not collecting outpatient prescriptions, there is a clear opportunity for misclassification. Second, they do not satisfactorily address the impact of Whitaker's observation that by starting the analysis at a particular point in time (e.g. 1996), then what patients received before this point is neither known nor accounted for. Similarly, they do not address the critique that their use of 'person years' in their studies tends to inflate the mortality in the 'off medication' group by disregarding the role of previous use of medication. This methodological approach would seem to transfer deaths attributable to the cumulative effects of years of exposure to antipsychotics into the 'off medication' group and presents a concern not addressed by Tiihonen and colleagues.

Third, the authors do not address Whitaker's observation that patients who received 0-6 months of antipsychotics have the lowest mortality risk in their own study (Tiihonen et al., 2009), but conclude that long-term use is associated with lower mortality.

Fourth, the RCT evidence on mortality cited in support of findings in naturalistic studies is possibly confounded by prior cessation of antipsychotics in patients allocated to placebo (common practice in these studies). That is to say that the observed higher mortality in this group may be a result of withdrawal effects (Herings \& Erkens, 2003). Such effects are known to include insomnia, restlessness, mood disturbances, severe psychotic relapse and akathisia, which can be persistent (Cerovecki et al., 2013; Horowitz, Murray, \& Taylor, 2020). This may explain why there is a spike in suicides in the months following abrupt cessation of antipsychotics (Herings \& Erkens, 2003). Moreover, the median duration of the studies captured in these meta-analyses was 6 weeks (IQR 4-10) (Schneider-Thoma et al., 2018) and 4 months (Khan, Faucett, Morrison, \& Brown, 2013), too short a time period for the metabolic effects of antipsychotics to manifest.

In addition to the above, support for their naturalistic findings from data from RCTs is selectively chosen. In the study by Khan and colleagues (Khan et al., 2013) the study authors are more cautious than Tiihonen and co-workers. They acknowledge that the data were not designed to evaluate mortality risk, that there was a considerable imbalance between treatment groups and that the short-term nature of the exposure to antipsychotics precluded firm conclusions. The study authors drew the more guarded conclusion that 'short-to-medium-term (3-4 months) exposure' to atypical antipsychotics is not associated with increased mortality risk. Further, the conclusions from the meta-analysis cited (Schneider-Thoma et al., 2018) are presented as conclusive when there was no significant difference found in mortality rates between drug and nondrug groups in schizophrenia (OR 0.69; 95\% CI 0.35-1.35). Moreover, the median duration of these studies was 6 weeks (IQR 4-10), too short a time period for the metabolic effects of antipsychotics to manifest. The conclusion that RCTs demonstrate a $30-50 \%$ reduction in mortality for those on medication is not strongly supported by the studies cited.

The treatment of schizophrenia has distinct pre- and post-neuroleptic eras - before and after the introduction of chlorpromazine in 1955. Mortality in psychotic illness was known 
to be elevated in the pre-neuroleptic era but this was felt largely to be due to an association between schizophrenia and tuberculosis (Lindelius \& Kay, 1973). Comparison of suicide rates is difficult because of the vast change in environment (and freedom to act) that people with schizophrenia have experienced over the last 60 years - from locked institutions to community care. What is clear is that the so-called mortality gap is still with us and that the size of this gap has hardly changed, at least in the last 30 years (Tanskanen, Tiihonen, \& Taipale, 2018). Whitaker and others raise the concern that not only is antipsychotic treatment not improving this situation, it may be actively contributing to this mortality gap. Very short-term RCTs and flawed observational studies are not reassuring in this respect. Longer-term placebo-controlled RCTs are required to definitively answer this pressing public health question.

\section{Conflict of interest}

DT reports personal fees from Lundbeck, Sunovion and Recordati and grants and personal fees from Janssen, outside the submitted work. MAH reports no conflicts of interest.

\section{References}

Cerovecki, A., Musil, R., Klimke, A., Seemüller, F., Haen, E., Schennach, R., \& Riedel, M. (2013). Withdrawal symptoms and rebound syndromes associated with switching and discontinuing atypical antipsychotics: Theoretical background and practical recommendations. CNS Drugs, 27 (7), 545-572. doi: 10.1007/s40263-013-0079-5.

Herings, R. M., \& Erkens, J. A. (2003). Increased suicide attempt rate among patients interrupting use of atypical antipsychotics. Pharmacoepidemiology and Drug Safety, 12(5), 423-424. doi: 10.1002/pds.837.

Horowitz, M. A., Murray, R. M., \& Taylor, D. (2020). Tapering antipsychotic treatment. JAMA Psychiatry. doi: 10.1001/jamapsychiatry.2020.2166.
Khan, A., Faucett, J., Morrison, S., \& Brown, W. A. (2013). Comparative mortality risk in adult patients with schizophrenia, depression, bipolar disorder, anxiety disorders, and attention-deficit/hyperactivity disorder participating in psychopharmacology clinical trials. JAMA Psychiatry, 70(10), 1091-1099. doi: 10.1001/jamapsychiatry.2013.149.

Lindelius, R., \& Kay, D. W. (1973). Some changes in the pattern of mortality in schizophrenia, in Sweden. Acta Psychiatrica Scandinavica, 49(3), 315-323. doi: 10.1111/j.1600-0447.1973.tb04425.x.

Ray, W. A., Chung, C. P., Murray, K. T., Hall, K., \& Stein, C. M. (2009). Atypical antipsychotic drugs and the risk of sudden cardiac death. New England Journal of Medicine, 360(3), 225-235. doi: 10.1056/NEJMoa0806994.

Salem, H., Nagpal, C., Pigott, T., \& Teixeira, A. L. (2017). Revisiting antipsychotic-induced akathisia: Current issues and prospective challenges. Current Neuropharmacology, 15(5), 789-798. doi: 10.2174/ $1570159 \times 14666161208153644$.

Schneider-Thoma, J., Efthimiou, O., Huhn, M., Krause, M., Reichelt, L., Röder, H., ... Leucht, S. (2018). Second-generation antipsychotic drugs and shortterm mortality: A systematic review and meta-analysis of placebo-controlled randomised controlled trials. The Lancet. Psychiatry, 5(8), 653-663. doi: 10.1016/s2215-0366(18)30177-9.

Tanskanen, A., Tiihonen, J., \& Taipale, H. (2018). Mortality in schizophrenia: 30-year nationwide follow-up study. Acta Psychiatrica Scandinavica, 138(6), 492-499. doi: 10.1111/acps.12913.

Taylor, D. M., Douglas-Hall, P., Olofinjana, B., Whiskey, E., \& Thomas, A. (2009). Reasons for discontinuing clozapine: Matched, case-control comparison with risperidone long-acting injection. British Journal of Psychiatry, 194(2), 165-167. doi: 10.1192/bjp.bp.108.051979.

Tiihonen, J., Lönnqvist, J., Wahlbeck, K., Klaukka, T., Niskanen, L., Tanskanen, A., \& Haukka, J. (2009). 11-year follow-up of mortality in patients with schizophrenia: A population-based cohort study (FIN11 study). Lancet (London, England), 374(9690), 620-627. doi: 10.1016/ s0140-6736(09)60742-x.

Tiihonen, J., Taipale, H., \& Correll, C. U. (2020). Commentary on Robert Whitaker's viewpoint. Psychological Medicine, 1-2. doi: 10.1017/ s0033291720003591.

Whitaker, R. (2020). Viewpoint: Do antipsychotics protect against early death? A critical view. Psychological Medicine, 1-10. doi: 10.1017/s003329172000358x. 